\title{
Acute ST-Segment Elevation Myocardial Infarction: Combined Intracoronary Low Dose Eptifibatide and Thrombus Aspiration
}

\author{
Sharaf Eldeen Mahmoud \\ Sohag University Hospital, Sohag, Egypt \\ Email: sharaf003@gmail.com
}

Received 26 August 2014; revised 9 October 2014; accepted 26 October 2014

Copyright (C) 2014 by author and Scientific Research Publishing Inc.

This work is licensed under the Creative Commons Attribution International License (CC BY). http://creativecommons.org/licenses/by/4.0/

(c) (i) Open Access

\section{Abstract}

Aim of the work: To evaluate the efficacy and safety of bolus only intra coronary platelet glycoprotein GP IIb/IIIa receptor antagonists combined with thrombus-aspiration during percutaneous coronary intervention (PCI) in ST-segment elevation myocardial infarction (STEMI). Patient and Methods: 25 consecutive STEMI patients treated by primary PCI including thrombus aspiration were prospectively enrolled, from a total of 180 PCIs performed at our institution between January 2013 and January 2014. All patients received aspirin (250 $\mathrm{mg}$ i.v.) and clopidogrel (600 or 300 mg if already on clopidogrel) plus standard heparin. Glycoprotein IIb/IIIa antagonists were administered at physicians' discretion before thrombus aspiration. Eptifibatide was used as a slandered Glycoprotein IIb/IIIa antagonists as a dose of the $180-\mu \mathrm{g} / \mathrm{kg}$ eptifibatide bolus only. The bolus was administered for 2 minutes via the thrombus aspiration catheter (STEMI cath of minvasys) just before thrombus aspiration. Twelve-lead ECGs were recorded at baseline and 60 min ( 45 - 75 min) after completion of PCI. The primary endpoint was the TIMI 3 patency of the infarctrelated coronary artery before PCI. Secondary endpoints were TIMI patency following PCI, ST resolution $60 \mathrm{~min}$ after PCI, all-cause death, reinfarction, urgent revascularization, stroke (haemorrhagic, non-haemorrhagic), and severe bleeding complications. Results: The angiography was performed in all 25 patients. The incidence of a TIMI flow grade 3 of the infarct-related coronary artery before PCI was $16 \%$ while TIMI grade 2 and I was $24 \%$ and $36 \%$ respectively. The incidence of a TIMI flow grade 0 (no reflow) of the infarct-related coronary artery before PCI was $24 \%$. Visible thrombus in $52 \%$ of patients and $40 \%$ of patients have more than one critically disease vessel. Primary PCI was performed in 25 patients. Eptifibatide intracoronary was given through thrombus aspiration catheter before thrombus aspiration. Drug eluting stents were implanted in $60 \%$ of patients. Only two patients with acute stent thrombosis did not receive any stents at PCI. One patient died $24 \mathrm{~h}$ after the procedure due to severe heart failure and cardiogenic shock. No reinfarction was noted within $\mathbf{3 0}$ days after the procedure. Generally heart failure improved significantly after the procedure and at the end of 30 day post operative. Only 3 patients had persistent heart 
failure class III (12\%). 84\% of patients had TIMI flow III at the end of procedure with over all significant improvement. Significant TIMI flow improvement after procedure was noticed with $P$ value, 001. Also the results showed significant shows of ST segment resolution after procedure. Conclusions: For patients with STEMI undergoing primary PCI, a routine combination of intracoronary administration of Eptifibatide before transcatheter thrombus aspiration is a safe procedure with low risk of hemorrhage and increases myocardial reperfusion, which ultimately improves outcomes. This therapeutic modality may be recommended for further improving myocardial reperfusion in patients with STEMI.

\section{Keywords}

Intra Coronary Platelet Glycoprotein GP II b/III a Receptor Antagonists, Acute Myocardial Infarction, Primary Percutaneous Intervention, Thrombus Aspiration Cathter

\section{Introduction}

In the sitting of acute ST-segment elevation myocardial infarction (STEMI), it has been constantly observed that, despite restoring good epicardial flow with PCI, myocardial perfusion at the cellular level remains impaired in nearly 50\% of STEMI patients. This led to the development of a new class of antiplatelet drugs, termed glycoprotein IIb/IIIa inhibitors (GPIs), which were hypothesized to be exquisitely effective in disaggregating the acute coronary thrombus responsible for STEMI. A recent meta-analysis including a little over 10,000 patients confirmed the concept that platelet inhibition beyond that provided by aspirin and thienopyridine with GPIs during elective percutaneous intervention resulted in reduced MI size without a significant increase in major bleeding [1]. The reduction in target vessel revascularization at 30 days was not significant, but there was a significant increase in the rate of minor bleeding (from $1.7 \%$ to $3 \%$ ) and thrombocyotopenia, but no increase in stroke.

Rapid achievement of reperfusion saves the myocardium at risk of ischemic injury. Despite advances in the reperfusion therapy there is substantial myocardial damage which may lead to heart failure. Indeed the Global Registry of Acute Coronary Events (GRACE) suggests that rates of heart failure are about 18\% in either ST elevation myocardial infarction or non ST elevation myocardial infarction. Primary percutaneous coronary intervention (PPCI) has become the treatment of choice for acute myocardial infarction (AMI) although there remains the same uncertainty about its net effect on the risk of heart failure as with thrombolytic therapy [2].

The (WEST) trial reported that myocardial damage leads to decreased left ventricular performance and increases heart failure after AMI in spite of timely reperfusion. The overall incidence of heart failure after acute myocardial infarction is almost $25 \%$ [3].

Recent advances to improve the outcomes of Acute Myocardial Infarction:

Device based strategies

Distal protection devices

Thrombectomy devices

Simple aspiration

Catheters

Pharmacologic therapy

Intracoronary/Intravenous

Streptokinase, Tenecteplase

Abciximab, Eptifibatide

Tirofiban

Adenosine

Remote ischemic

Preconditioning

Postconditioning

Cyclosporine 
Erythropoietin

Cardiac repair or

Regeneration with

Stem cell therapy

Suboptimal myocardial salvage despite quick reperfusion therapy is a matter of current research and new strategy is being tested to minimize myocardial loss without increasing the risk of hemorrhage.

This strategy consists of injecting a low dose of Eptifibatide intracoronary through the thrombus aspiration catheter just before thrombus aspiration.

\subsection{Thrombus Aspiration during PPCI}

The distal embolization of thrombus leads to no reflow phenomenon, leading to larger infarct size and ultimately reduced survival [4].

Distal protection devices, thrombectomy devices and thrombus aspiration catheters have been used to improve the outcome of PCI. They have reduced distal embolization and resulted in enhanced TIMI grade 3 flow and more complete ST-segment resolution. The meta-analysis of the clinical trials investigating these devices showed that the simple aspiration catheters were more effective than the mechanical thrombectomy devices or distal protection devices. The thrombus aspiration catheters have been able to reduce one year mortality and reduced the combined end point of any death, myocardial infarction or target vessel revascularization [5].

The results of Thrombus Aspiration Compared to Balloon Angioplasty (TAPAS) trial have been reported that adjunctive aspiration resulted in enhanced rates of normal angiographic myocardial perfusion (blush) and STsegment resolution. At 30 days, mortality tended to be less in patients treated with thrombus aspiration [6].

\subsection{Intracoronary Gp IIb/IIIa Inhibitors}

Intracoronary administration of drugs increases local drug concentration several fold. The increased concentration of Gp IIb/IIIa inhibitors like Abciximab, Eptifibatide, Tirofiban are shown to improve the outcomes of PCI safely and efficaciously in terms of reduction in infarct size, peri-procedural MI and improved TIMI flow [7].

(The Comparison of Intracoronary Versus Intravenous Abciximab Administration during Emergency Reperfusion of ST-Segment Elevation Myocardial Infarction) Trial. No difference was noted in ST resolution between the intracoronary versus intravenous Abciximab groups. However, intracoronary administration was associated with improved myocardial perfusion assessed by myocardial blush grade and a smaller enzymatic infarct size [8].

Similarly, in the Intracoronary Eptifibatide (ICE) Trial, the intracoronary bolus administration of eptifibatide during PCI in patients with acute coronary syndromes resulted in higher local platelet glycoprotein IIb/IIIa recept or occupancy, which was associated with improved microvascular perfusion demonstrated by an improved corrected TIMI Frame Count [9].

\section{Aim of the Work}

To evaluate the efficacy and safety of bolus only intra coronary platelet glycoprotein GP II b/III a receptor antagonists combined with thrombus-aspiration during percutaneous coronary intervention (PCI) in ST-segment elevation myocardial infarction (STEMI).

\section{Patient and Methods}

25 consecutive STEMI patients treated by primary PCI including thrombus aspiration were prospectively enrolled, from a total of 180 PCIs performed at our institution between January 2013 and January 2014.

All patients were admitted to our coronary care unit with chest pain, new persistent ST-segment elevation, cardiac troponin $\mathrm{T}(\mathrm{TnT})$ elevation, and/or new regional wall motion abnormalities. Exclusion criteria for all patients were: age $>80$ years, infarction secondary to ischemia due to an imbalance of $\mathrm{O}_{2}$ supply and demand, previous ECG abnormalities that could prevent the recognition of ST-segment shift, recent or chronic infective or inflammatory diseases, malignancy, surgery or trauma in the previous month, or coronary anatomy judged unfavorable for thrombus aspiration. Patients undergoing rescue PCI were also excluded.

All patients received aspirin (250 mg i.v.) and clopidogrel (600 or $300 \mathrm{mg}$ if already on clopidogrel) plus 
standard heparin to maintain an activated clotting time of $>300 \mathrm{~s}$.

Glycoprotein IIb/IIIa antagonists were administered at physicians' discretion before thrombus aspiration. Local Ethics Committee approved the study and all patients signed an informed consent.

Eptifibatide was used as a slandered Glycoprotein IIb/IIIa antagonist as a dose of The $180-\mu \mathrm{g} / \mathrm{kg}$ eptifibatide bolus was drawn from $20 \mathrm{mg}$ in a $10-\mathrm{mL}$ vial of eptifibatide and was diluted with sterile water. The bolus was administered for 2 minutes via the thrombus aspiration catheter (STEMI cath of minvasys) just before thrombus aspiration.

Catheter for thrombo-aspiration in acute ST-Elevation Myocardial Infarction of Minvasys with the following criteria, Single radiopaque marker/Single-operator device/Currently the highest ratio ID/OD.

\subsection{ST-Segment Resolution Evaluation}

Twelve-lead ECGs were recorded at baseline and 60 min (45 - 75 min) after completion of PCI. The sum of the ST-segment elevation was measured by a central core laboratory without knowledge of treatment assignment. Complete resolution was defined as resolution of the initial sum of ST-segment elevation $\geq 70 \%$. Partial resolution was defined as ST resolution $<70 \%-30 \%$, whereas no resolution was defined as ST resolution $<30 \%$. In addition, the mean extent of ST resolution was calculated and the rate of patients with $\geq 50 \%$ ST resolution was evaluated [10].

\subsection{Angiographic Analysis}

Angiograms were evaluated centrally and blinded. Patency of the infarct-related artery was evaluated centrally and blinded according to the TIMI criteria. The myocardial perfusion grade was assessed as described following the criteria of the TIMI group [11].

The primary endpoint was the TIMI 3 patency of the infarct-related coronary artery before PCI. Secondary endpoints were TIMI patency following PCI, ST resolution 60 min after PCI, all-cause death, reinfarction, urgent revascularization, stroke (haemorrhagic, non-haemorrhagic), and severe bleeding complications.

Reinfarction was defined by recurrent signs and symptoms of ischaemia at rest accompanied by new or recurrent ST-segment elevations of $\geq 0.1 \mathrm{mV}$ in at least two contiguous leads lasting $\geq 30 \mathrm{~min}$ and/or enzyme/biochemical evidence of reinfarction: re-elevation of CK-MB or troponin to above the upper limit of normal and increased by $\geq 50 \%$ over the previous value.

Severe bleeding complications were defined as bleeds that lead to haemodynamic compromise requiring intervention (e.g. blood or fluid replacement, inotropic support, surgical repair) or life-threatening or fatal bleeds, and intracerebral bleedings. Urgent revascularization was defined as recurrent chest pain despite optimized medical therapy leading to an unscheduled revascularization procedure. Stroke was diagnosed based on an imaging study and an expert neurologist opinion. Clinical follow-up for all secondary endpoints was done for 30 days after the index event.

Troponin $\mathrm{T}$ and creatinine kinase (CK) levels were obtained on admission. CK values were obtained every 8 $12 \mathrm{~h}$ during the first $48 \mathrm{~h}$ and the peak level was recorded.

Descriptive statistics was generated for baseline and clinical demographics, treatment variables, and outcomes. A P-value of $<0.05$ was considered significant. The analysis was performed on an intention-to-treat basis. The test for the primary endpoint was two-sided and not adjusted for multiple testing. This was done with the Fisher's exact test after dichotomizing the TIMI patency rates. All other comparisons were performed two-sided and the P-values have to be interpreted in a descriptive sense.

Killip class I includes individuals with no clinical signs of heart failure.

Killip class II includes individuals with rales or crackles in the lungs, an S3, and elevated jugular venous pressure. Killip class III describes individuals with frank acute pulmonary edema. Killip class IV describes individuals in cardiogenic shock or hypotension (measured as systolic blood pressure lower than $90 \mathrm{mmHg}$ ), and evidence of peripheral vasoconstriction (oliguria, cyanosis or sweating).

TIMI 0 flow (no perfusion) refers to the absence of any antegrade flow beyond a coronary occlusion. TIMI 1 flow (penetration without perfusion) is faint antegrade coronary flow beyond the occlusion, with incomplete filling of the distal coronary bed. TIMI 2 flow (partial reperfusion) is delayed or sluggish antegrade flow with complete filling of the distal territory. TIMI 3 is normal flow which fills the distal coronary bed completely. 


\section{Results}

\subsection{Baseline Data and Angiographic Findings}

Between January 2013 and January 2014, 25 consecutive STEMI patients treated by primary PCI including thrombus aspiration and administration of intracoronary Eptifibatide. Patient baseline characteristics are summarized in Table 1.

Mean age was 51 year and 60\% of patients had anterior MI, incidence of DM and Hyperlipidaemia was $48 \%$ and $68 \%$ respectively. Patients with Killip class $>1$ represents $44 \%$.

The angiography was performed in all 25 patients, The angiographic findings are summarized in Table 2 . The incidence of a TIMI flow grade 3 of the infarct-related coronary artery before PCI was 16\% while TIMI grade 2 and I was 24\% and 36\% respectively. The incidence of a TIMI flow grade 0 (no reflow) of the infarct-related coronary artery before PCI was $24 \%$. Visible thrombus in $52 \%$ of patients and $40 \%$ of patients have more than one critically disease vessel.

Detailed analysis of the data suggests that those patients with the highest risk.

Table 1. Patient baseline characteristics.

\begin{tabular}{cc} 
Mean age (years) & $51 \pm 12$ \\
Male sex & $56 \%$ \\
Presenting characteristics & \\
HTN & $36 \%$ \\
Anterior infarct location & $60 \%$ \\
Killip class >1 & $44 \%$ \\
Medical history & \\
Smoker & $51 \%$ \\
Hyperlipidaemia & $64 \%$ \\
Diabetes mellitus & $48 \%$ \\
Prior IHD & $28 \%$ \\
Prior PCI & $12 \%$ \\
Prior CABG & $4 \%$ \\
\hline
\end{tabular}

Table 2. Angiographic data.

Infarct vessel

$\begin{array}{cc}\text { Left main } & 1(4 \%) \\ \text { LAD } & 14(56 \%) \\ \text { CX } & 4(16 \%) \\ \text { RCA } & 6(24 \%)\end{array}$

Bypass graft

0

Diseased vessels

1

$15(60 \%)$

more than 1

$10(40 \%)$ 


\subsection{PCI Results}

Primary PCI was performed in 25 patients. Eptifibatide intracoronary was given through thrombus aspiration catheter before thrombus aspiration. Drug eluting stents were implanted in $60 \%$ of patients. Summary of procedural data are shown in Table 3.

\subsection{Post Procedural Data}

Summary of post procedural data are illustrated in Table 4.

The final TIMI flow grade in patients with PCI is shown in Figure 1.

One patient died $24 \mathrm{~h}$ after the procedure due to severe heart failure and cardiogenic shock. No reinfarction was noted within 30 days after the procedure. Generally heart failure improved significantly after the procedure and at the end of 30 day post operative. Only 3 patients had persistent heart failure class III (12\%). 84\% of patients had TIMI flow III at the end of procedure with over all significant improvement. Figure 2 illustrates also

Table 3. Procedural data.

\begin{tabular}{cccc}
\hline Number of target lesions & One lesion & Two lesions & Three lesions \\
& $64 \%$ & $32 \%$ & $3 \%$ \\
Stent diameter & $2.75 \mathrm{~mm}$ & $3 \mathrm{~mm}$ & $3.5 \mathrm{~mm}$ \\
Number of stents & $24 \%$ & 2 & $40 \%$ \\
& 1 & $24 \%$ & $4 \%$ \\
\hline
\end{tabular}

Note/Only two patients with acute stent thrombosis did not receive any stents at PCI.

Table 4. Summary of post procedural results.

\begin{tabular}{|c|c|c|c|c|}
\hline \multirow{2}{*}{$\begin{array}{l}\text { TIMI FLOW at end } \\
\text { of procedure }\end{array}$} & Grade 0 & Grade 1 & Grade II & Grade III \\
\hline & $0 \%$ & $4 \%$ & $12 \%$ & $84 \%$ \\
\hline \multirow{2}{*}{$\begin{array}{l}\text { ST segment resolution } \\
\text { after } 1 \text { hour of PCI }\end{array}$} & No resolution & Partial resolution & \multicolumn{2}{|c|}{ Complete resolution } \\
\hline & $12 \%$ & $40 \%$ & \multicolumn{2}{|l|}{$48 \%$} \\
\hline \multirow{2}{*}{ Bleeding } & Minor bleeding & Major bleeding & \multirow{2}{*}{\multicolumn{2}{|c|}{$\begin{array}{l}\text { One patient had minor cerebral hemorrhage and } \\
\text { hemi paresis with complete improvement } \\
\text { and no residual deficit after } 1 \mathrm{~W} \text {. }\end{array}$}} \\
\hline & $24 \%$ & $4 \%$ & & \\
\hline
\end{tabular}

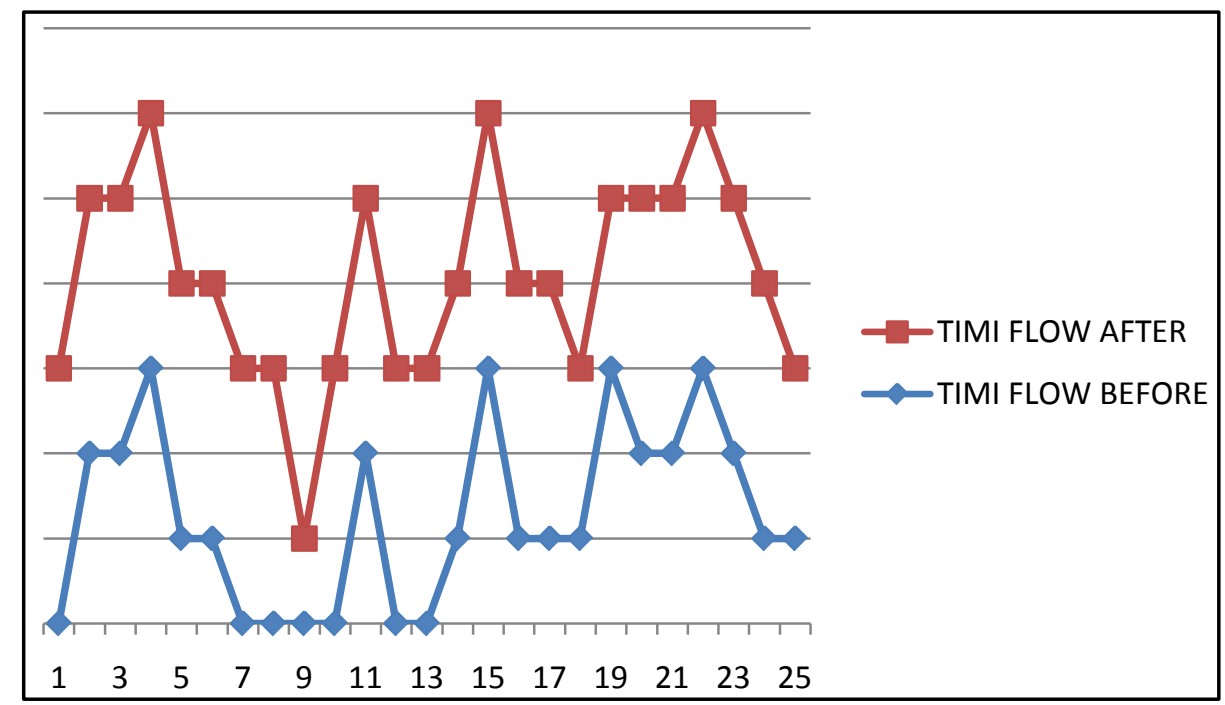

Figure 1. TIMI flow improvement after procedure P value, 001. 


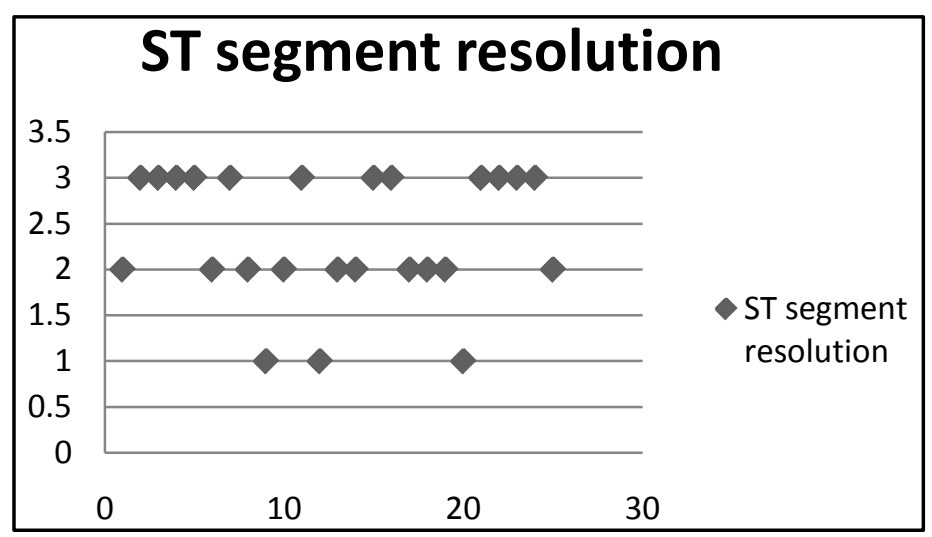

Figure 2. ST segment resolution after procedure in which 3 complete resolution and 1 no resolution.

ST segment resolution after procedure in which 3 complete resolution and 1 no resolution. Figures 3-5 demonstrate some interesting cases performed during the study, and finally Figure 6 shows the CT brain of one patient with non fatal intra cranial hemorrhage.

\section{Discussion}

In patients with acute myocardial infarction restoration of epicardial blood flow has been shown to be the important predictor of clinical and angiographic outcome. The goal of reperfusion therapy should be to restore not only epicardial patency but also improving myocardial tissue perfusion [12]. Myocardial blush grade is an important predictor of infarct size and survival. It is also a strong angiographic predictor of mortality in patients with TIMI3 flow after angioplasty [13].

Surprisingly, there are very limited data on the combined application of IC GPIs and manual thrombectomy in STEMI patients [14].

The randomized Comparison of intracoronary versus intravenous abciximab administration during emergency reperfusion of ST-segment elevation myocardial infarction (CICERO) trial is one of the very few trials comparing the effects of IC versus IV administration of abciximab in STEMI patients undergoing primary PCI with manual thrombosuction.

An interesting case report described the management of no reflow accompanied by marked hypotension and ST-segment elevation in a patient undergoing PCI for a left circumflex lesion. The catastrophe was promptly corrected with IC tirofiban given at a dose of $10 \mu \mathrm{g} / \mathrm{kg}$. 32 Another case report suggested that IC administration of tirofiban can be safely employed to treat acute stent thrombosis occurring in a left anterior descending artery stent deployment. A bare-metal stent measuring $3.5 \times 18 \mathrm{~mm}$ was deployed in a 46-year-old male patient presenting with STEMI and there was immediate stent thrombosis leading to severe chest pain and shock. An IC bolus of tirofiban $(10 \mu \mathrm{g} / \mathrm{kg})$ was able to quickly achieve good antegrade flow with resolution of symptoms and haemodynamic compromise when repeated attempts with balloon inflation did not succeed [15].

A further case report describes a 50-year-old male who had undergone primary PCI for acute anterior STEMI being readmitted with subacute stent thrombosis owing to inadvertently missing his aspirin dose for almost a week. His angiogram revealed tight subtotal stenosis and large thrombus burden within the sirolimus-eluting stent that had been deployed previously in his proximal left anterior descending coronary artery. Brisk antegrade flow was achieved with a $25 \mu \mathrm{g} / \mathrm{kg}$ bolus of IC tirofiban and subsequent plain balloon inflations [16].

In a previous trial done, to evaluate the impact of Export Aspiration Catheter with restoration of ECG changes, arterial flow and myocardial perfusion in patients with ST segment elevation Myocardial Infarction undergoing Percutaneous Coronary Intervention (PCI). The results of this study showed that thrombus aspiration with export catheter is feasible with STEMI and improves angiographic and ECG changes in which TIMI3 flow in thrombus aspiration group was $75 \%$ at the end of PCI procedure in our study reached $84 \%$ and as regard ST segment resolution was the same $47 \%$ versus $48 \%$ in our study [17].

Another study [18] showed results of TIMI flow 3 equal to our results but ECG resolution was achieved in less than $40 \%$ of patients in spite that more than $50 \%$ received GP IIb IIIa inhibitors IV. No reflow in $14 \%$. 

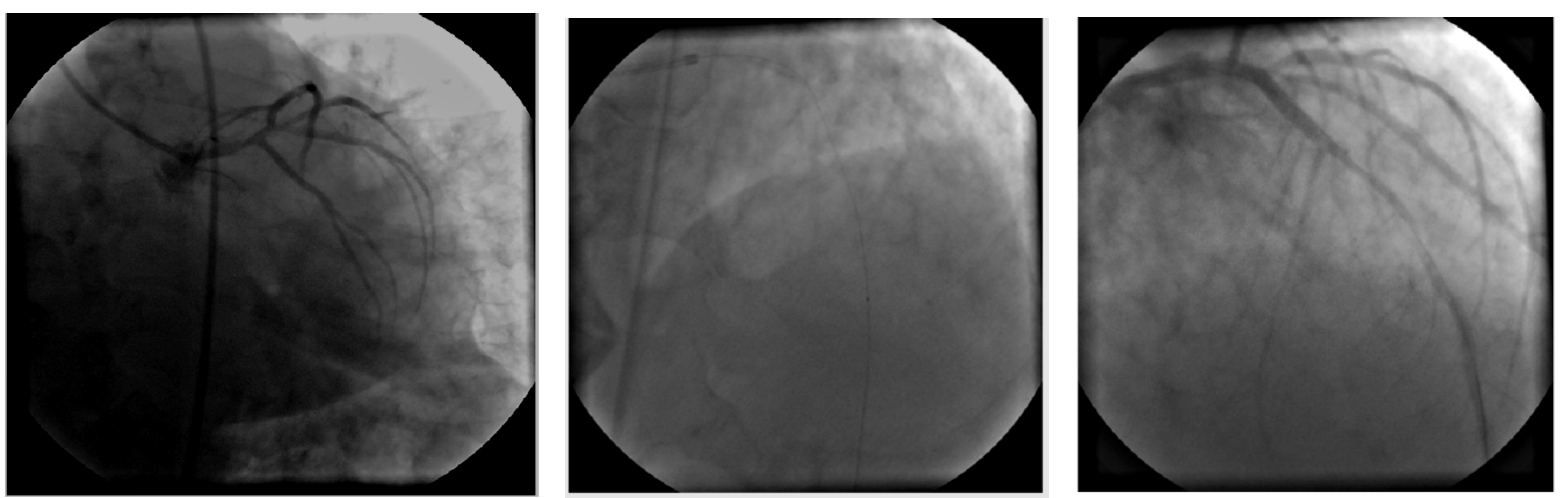

Figure 3. Shows LM and mid LAD thrombus, thrombus aspiration, eptifipatide IC and LM stenting.
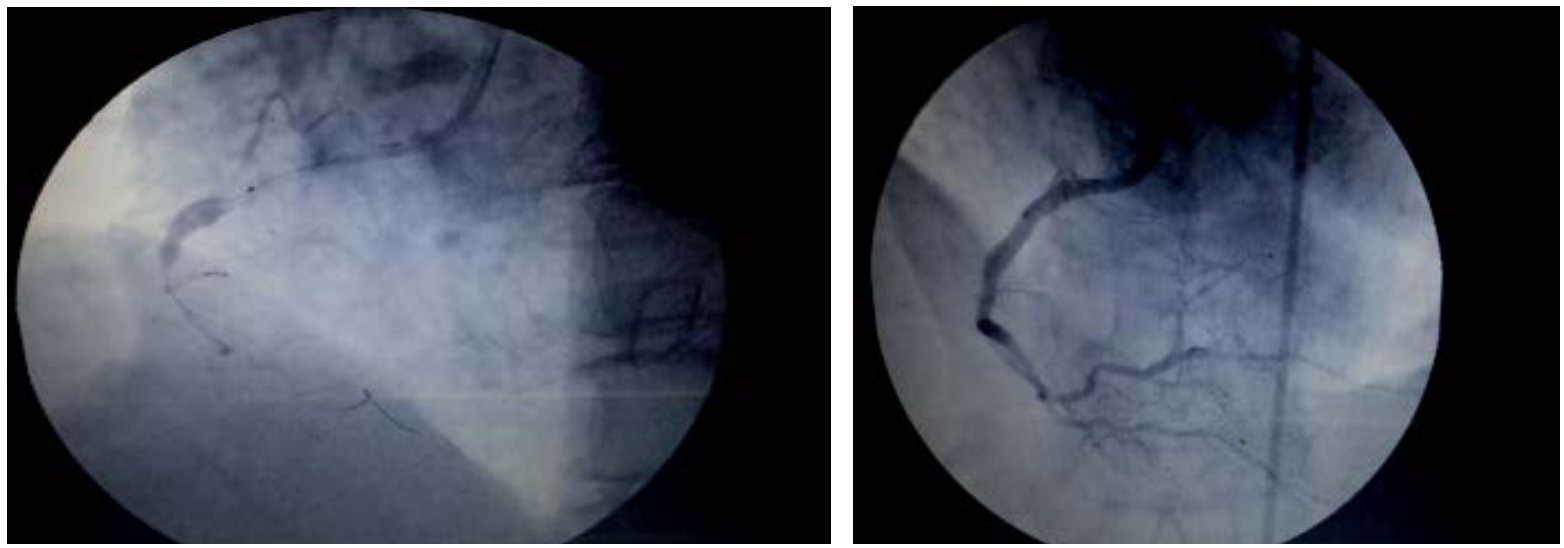

Figure 4. RCA shows large thrombus, thrombus aspiration, eptifipatide IC and stenting.
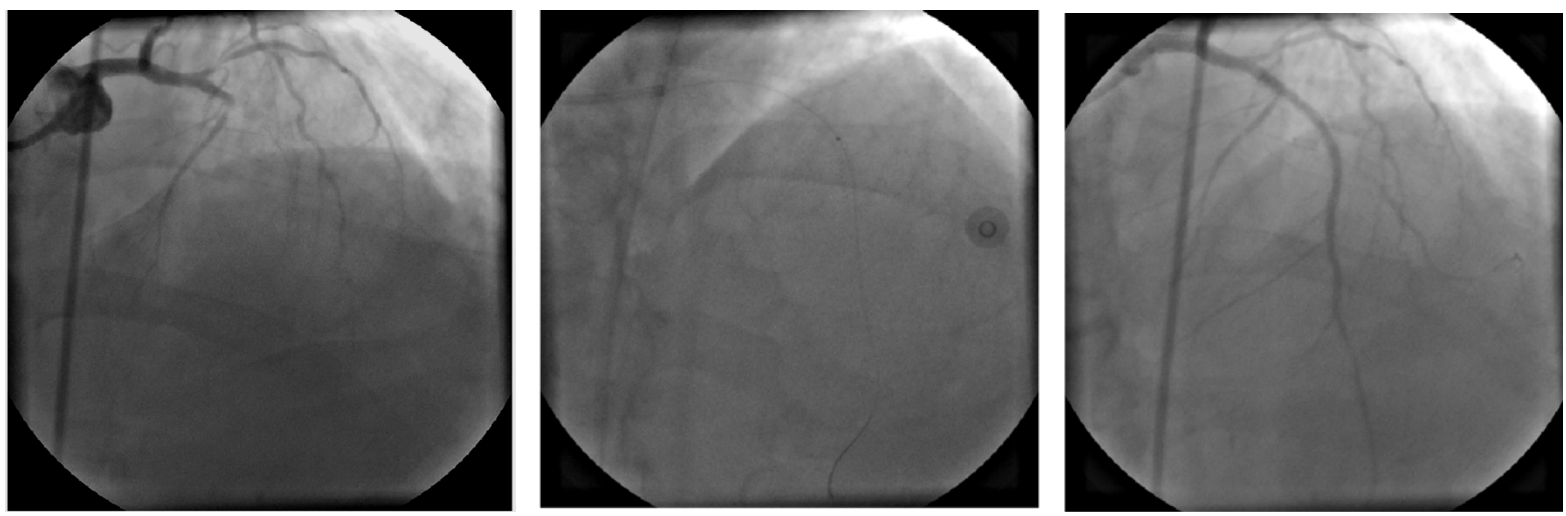

Figure 5. Shows stent thrombosis of LAD and TIMI flow III after procedure.

In: VAMPIRE (VAcuuM as PIration thrombus REmoval) Trial FREE [19] St resolution 40\% and no reflow $12 \%$ while in our study 0 to one TIMI flow around $4 \%$.

From which we can conclude higher efficacy of combination therapy over thrombectomy alone specially in resolution of ST segment and prevention of no reflow.

Affection of one patient by non fatal major bleeding in 25 cases assumes safety of the technique. No cases of thrombocytopenia were recorded.

\section{Conclusion}

For patients with STEMI undergoing primary PCI, a routine combination of intracoronary administration of Ep- 


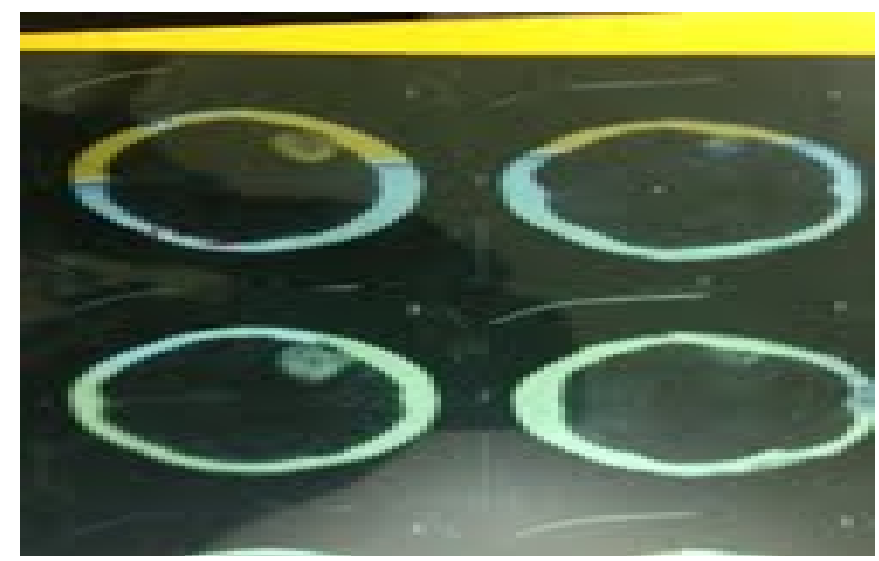

Figure 6. CT brain without contrast shows non fatal intracranial hemorrhage in one patient.

tifibatide before transcatheter thrombus aspiration is a safe procedure with low risk of hemorrhage and increases myocardial reperfusion, which ultimately improves outcomes. This therapeutic modality may be recommended for further improving myocardial reperfusion in patients with STEMI.

\section{Limitations}

Larger randomized multicenter trials associated with head to head comparison with IV Eptifibatide are needed for further assessment of higher efficacy and better safety of this technique.

\section{References}

[1] Winchester, D.E., Wen, X., Brearley, W.D., et al. (2011) Efficacy and Safety of Glycoprotein IIb/IIIa Inhibitors during Elective Coronary Revascularization: A Meta-Analysis of Randomized Trials Performed in the Era of Stents and Thienopyridines. Journal of the American College of Cardiology, 57, 1190-1199. http://dx.doi.org/10.1016/j.jacc.2010.10.030

[2] Steg, P.G., Dabbous, O.H., Feldman, L.J., Cohen-Solal, A., Aumont, M.C., López-Sendón, J., Budaj, A., Goldberg, R.J., Klein, W. and Anderson Jr., F.A. (2004) Global Registry of Acute Coronary Events Investigators. Determinants and Prognostic Impact of Heart Failure Complicating Acute Coronary Syndromes: Observations from the Global Registry of Acute Coronary Events (GRACE). Circulation, 109, 494-499. http://dx.doi.org/10.1161/01.CIR.0000109691.16944.DA

[3] Armstrong, P.W. (2006) A Comparison of Pharmacologic Therapy with/without Timely Coronary Intervention vs Primary Percutaneous Intervention Early after ST-Elevation Myocardial Infarction: The WEST (Which Early ST-Elevation Myocardial Infarction Therapy) Study. European Heart Journal, 27, 1530-1538. http://dx.doi.org/10.1093/eurheartj/ehl088

[4] Stone, G.W. (2008) Angioplasty Strategies in ST-Segment-Elevation Myocardial Infarction: Part II: Intervention After Fibrinolytic Therapy, Integrated Treatment Recommendations, and Future Directions. Circulation, 118, 552-566. http://dx.doi.org/10.1161/CIRCULATIONAHA.107.739243

[5] Singh, M., Reeder, G.S., Ohman, E.M., et al. (2001) Does the Presence of Thrombus Seen on a Coronary Angiogram Affect the Outcome after Percutaneous Coronary Angioplasty? An Angiographic Trials Pool Data Experience. Journal of the American College of Cardiology, 38, 624-630. http://dx.doi.org/10.1016/S0735-1097(01)01445-0

[6] Vlaar, P.J., Svilaas, T., van der Horst, I.C., Diercks, G.F.H., Fokkema, M.L., de Smet, B.J.G.L., van den Heuvel, A.F.M., Anthonio, R.L., Jessurun, G.A., Tan, E., Suurmeijer, A.J.H. and Zijlstra, F. (2008) Cardiac Death and Reinfarction after 1 Year in the Thrombus Aspiration during Percutaneous Coronary Intervention in Acute Myocardial Infarction Study (TAPAS): A 1-Year Follow-Up Study. Lancet, 371, 1915-1920. http://dx.doi.org/10.1016/S0140-6736(08)60833-8

[7] Gibson, C.M., Zorkun, C. and Kunadian, V. (2008) Intracoronary Administration of Abciximab in ST-Elevation Myocardial Infarction. Circulation, 118, 6-8. http://dx.doi.org/10.1161/CIRCULATIONAHA.108.780718

[8] Thiele, H., Schindler, K., Friedenberger, J., et al. (2008) Intracoronary Compared with Intravenous Bolus Abciximab Application in Patients with ST-Elevation Myocardial Infarction Undergoing Primary Percutaneous Coronary Inter- 
vention: The Randomized Leipzig Immediate Percutaneous Coronary Intervention Abciximab IV Versus IC in STElevation Myocardial Infarction Trial. Circulation, 118, 49-57.

http://dx.doi.org/10.1161/CIRCULATIONAHA.107.747642

[9] Ten Berg, J.M., van’t Hof, A.W.J., Dill, T., et al. (2010) Effect of Early, Pre-Hospital Initiation of High Bolus Dose Tirofiban in Patients with ST-Segment Elevation Myocardial Infarction on Short- and Long-Term Clinical Outcome (ON TIME 2). Journal of the American College of Cardiology, 55, 2446-2455. http://dx.doi.org/10.1016/j.jacc.2009.11.091

[10] Schröder, R., Zeymer, U., Wegscheider, K. and Neuhaus, K.L. (1999) Comparison of the Predictive Value of ST Segment Elevation Resolution 90 and 180 Minutes after Start of Streptokinase in Acute Myocardial Infarction. A Substudy of the Hirudin for Improvement of Thrombolysis (HIT)-4 Study. European Heart Journal, 20, 1563-1571. http://dx.doi.org/10.1053/euhj.1999.1664

[11] Gibson, C.M., Cannon, C.P., Murphy, S.A., Ryan, K.A., Mesley, R., Marble, S.J., Mc Cabe, C.H., van De Werf, F. and Braunwald, E. (2000) The Relationship of the TIMI Myocardial Perfusion Grade to Mortality Following Thrombolytic Administration. Circulation, 101, 125-130. http://dx.doi.org/10.1161/01.CIR.101.2.125

[12] Roe, M.T., Ohman, E.M., Maas, A.C., Christenson, R.H., Mahaffey, K.W., Gronger, C.B., et al. (2001) Shifting the Open Artery Hypothesis Downstream: The Quest for Optimal Reperfusion. Journal of the American College of Cardiology, 37, 9-18. http://dx.doi.org/10.1016/S0735-1097(00)01101-3

[13] Cannon, C.P. (2001) Importance of TIMI3 Flow. Circulation, 104, 624-626. http://dx.doi.org/10.1161/01.CIR.104.6.624

[14] Natarajan, D. (2011) Combined Intracoronary Glycoprotein Inhibitors and Manual Thrombus Extraction in Patients with Acute ST-Segment Elevation Myocardial Infarction-Does Incorporation of Both Have a Legitimate Role? Interventional Cardiology, 6, 182-185.

[15] Erden, I., Ozhan, H. and Ordu, S. (2010) A Case of Stent Thrombosis Treated Successfully with Intracoronary Tirofiban. Kardiologia Polska, 68, 485-487.

[16] Natarajan, D., Mukherjee, N., Yadav, A., et al. (2010) Intracoronary Tirofiban with Plain Balloon Angioplasty for Subacute Stent Thrombosis. http://www.tctmd.com

[17] Ashraf, T., Rasool, S.I., Saghir, T., Rizvi, S.N., Qamar, N., Zaman, K.S., Ishaque, M. and Kundi, A. (2007) Aspiration of Thrombus in ST Segment Elevation Myocardial Infarction. Journal of Pakistan Medical Association, 57, 359-362.

[18] De Vita, M., Burzotta, F., Porto, I., Dudek, D., Lefèvre, T., Trani, C., Mielecki, W., Niccoli, G., Biondi-Zoccai, G.G. and Crea, F. (2010) Thrombus Aspiration in ST Elevation Myocardial Infarction: Comparative Efficacy in Patients Treated Early and Late after Onset of Symptoms. Heart, 96, 1287-1290. http://dx.doi.org/10.1136/hrt.2009.184341

[19] Ikari, Y., Sakurada, M., Kozuma, K., Kawano, S., Katsuki, T., Kimura, K., Suzuki, T., Yamashita, T., Takizawa, A., Misumi, K., Hashimoto, H. and Isshiki, T., VAMPIRE Investigators. (2008) Upfront Thrombus Aspiration in Primary Coronary Intervention for Patients with ST-Segment Elevation Acute Myocardial Infarction: Report of the VAMPIRE (VAcuuM asPIration thrombus REmoval) Trial. JACC-Cardiovascular Interventions, 1, 424-431. 
Scientific Research Publishing (SCIRP) is one of the largest Open Access journal publishers. It is currently publishing more than 200 open access, online, peer-reviewed journals covering a wide range of academic disciplines. SCIRP serves the worldwide academic communities and contributes to the progress and application of science with its publication.

Other selected journals from SCIRP are listed as below. Submit your manuscript to us via either submit@scirp.org or Online Submission Portal.
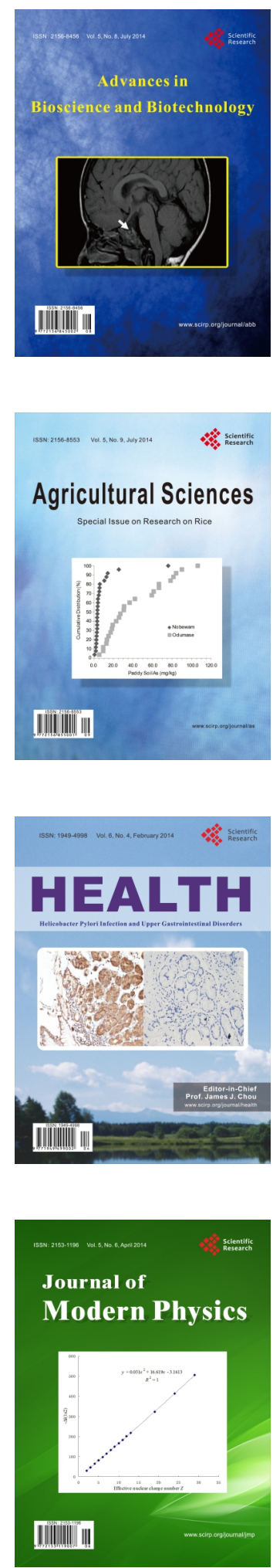
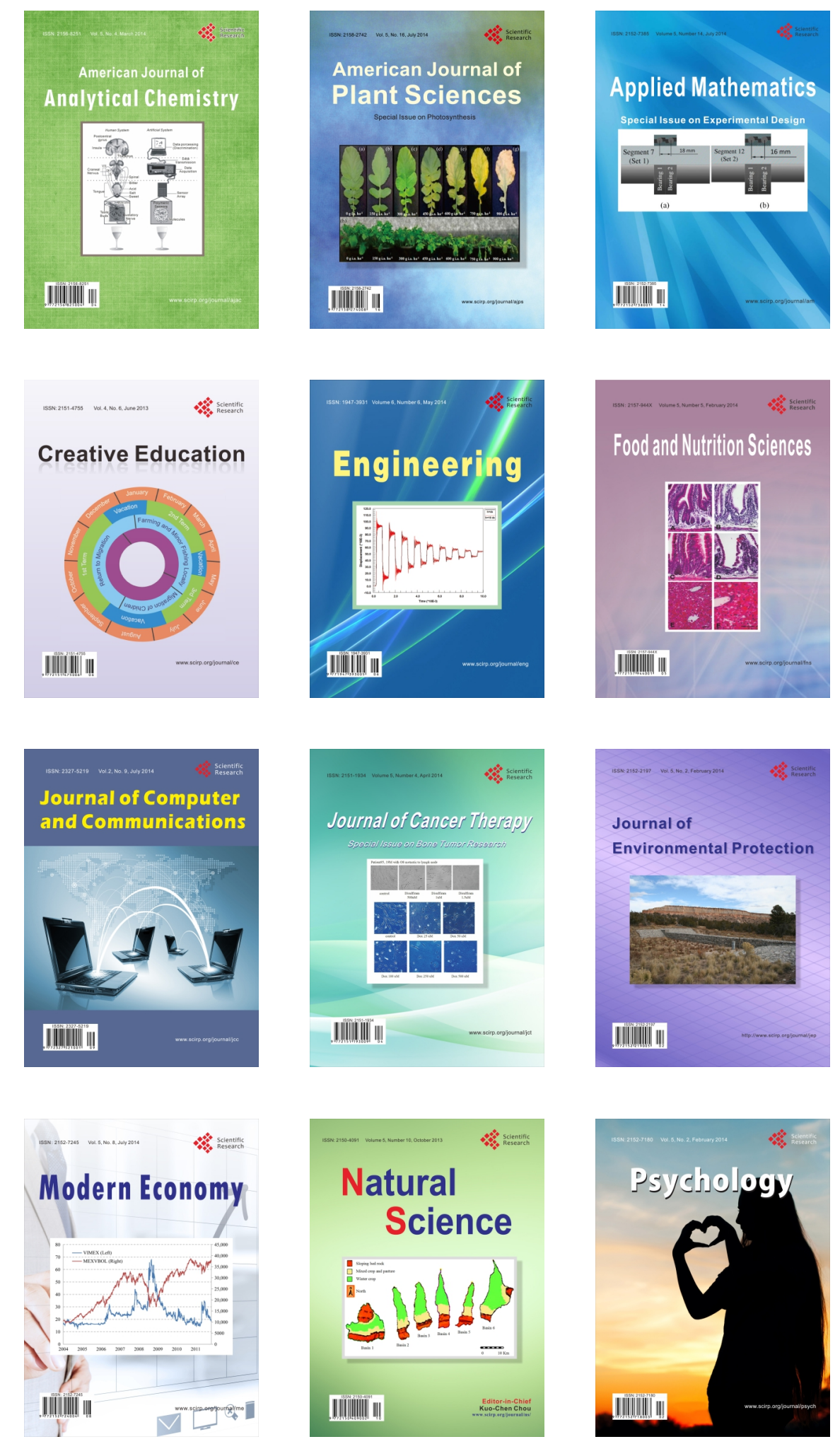